\section{Realizing the Full Potential of PET for Measuring the Biodistribution of Novel Anticancer Agents}

TO THE EDITOR: The unique specificity and sensitivity of PET offers a means to support the development of new anticancer agents. Radiolabeling of a drug with ${ }^{11} \mathrm{C}$ or ${ }^{18} \mathrm{~F}$ allows PET measurement of drug biodistribution to be undertaken at the microdose and therapeutic dose level (1-3). An example is targeted agents such as antisense oligonucleotides, for which in vivo biodistribution was first reported using PET in primates in 1998 (4).

Since this groundbreaking work in PET-based molecular imaging, there have been few follow-up studies on human cancer patients. However, a recent report on the use of an ${ }^{11} \mathrm{C}$-labeled second-generation antisense oligonucleotide to survivin (5) demonstrated the power of the PET technology for theranostics drug development (6). Not only was the biodistribution of the novel agent shown, but an important dose effect was demonstrated, with implications on how such an agent is administered to patients (6).

This latest study highlights several important questions on how PET-based molecular imaging can affect cancer theranostics drug development in the future:

- Why has it taken so long to translate from the earlier pioneering preclinical work in PET to human studies?

- Why are more studies not being undertaken?

- Why has PET underachieved in making an impact on cancer drug development and theranostics, to which it potentially can make a major unique contribution?

- Can these studies be undertaken only within the expensive and complex facilities of "big science PET centers," resulting in the study of fewer than ideal numbers of patients?

- Why are studies not undertaken in specialized oncology departments, where important contributions and proposals for clinical experimental studies arise?

We believe the answers to these challenging questions rest on the fact that the advanced technology required is too complex and expensive for leading clinical researchers to access easily or productively. There is still insufficient access to the generic radiolabeling methodologies available to exploit the wealth of opportunities for PET studies of biologic macromolecules. A paradigm shift in drug development, to bring PET into mainstream clinical translational research and to enhance chemistry and biology support, is also clearly required. We write to draw your readers' attention to potential solutions: the development and availability of low-cost, low-radiation-emitting, simple-to-operate positron-emitting radioisotope generators and of inexpensive disposable, good-manufacturing-practice-compliant "card-based" radiosynthesis platforms that lighten regulatory overload (7). These systems should be used to implement generic radiolabeling procedures, such as those reported for peptides and proteins (8) and oligonucleotides (9). We hope the development of these new technologies is encouraged by others in the molecular imaging community.

\section{REFERENCES}

1. Saleem A, Harte RJ, Matthews JC, et al. Pharmacokinetic evaluation of $\mathrm{N}$-[2-(dimethylamino)ethyl]acridine-4-carboxamide in patients by positron emission tomography. J Clin Oncol. 2001;19:1421-1429.

2. Aboagye EO, Price PM, Jones T. In vivo pharmacokinetics and pharmacodynamics in drug development using positron emission tomography. Drug Discov Today. 2001;6:293-302.

3. Bergström M, Grahnén A, Långström B. Positron emission tomography microdosing: a new concept with application in tracer and early clinical drug development. Eur J Clin Pharmacol. 2003;59:357-366.

4. Tavitian B, Terrazzino S, Kühnast B, et al. In vivo imaging of oligonucleotides with positron emission tomography. Nat Med. 1998;4:467-471.

5. Talbot DC, Ranson M, Davies J, et al. Tumor survivin is downregulated by the antisense oligonucleotide LY2181308: a proof-of-concept, first-in-human dose study. Clin Cancer Res. 2010;16:6150-6158.

6. Saleem A, Matthews JC, Ranson M, et al. Molecular imaging and pharmacokinetic analysis of carbon-11 labeled antisense oligonucleotide LY2181308 in cancer patients. Theranostics. 2011;1:290-301.

7. Långström B, Grahnen A, Honoré $\mathrm{PH}$, et al. The risk of exaggerated risk aversion: a life and death struggle for molecular imaging. Eur J Nucl Med Mol Imaging. 2009;36:1693-1694.

8. de Bruin B, Kuhnast B, Hinnen F, et al. 1-[3-(2-[ $\left.{ }^{18} \mathrm{~F}\right]$ fluoropyridin-3-yloxy)propyl] pyrrole-2,5-dione: design, synthesis, and radiosynthesis of a new $\left[{ }^{18} \mathrm{~F}\right]$ fluoropyridine-based maleimide reagent for the labeling of peptides and proteins. Bioconjug Chem. 2005;16:406-420.

9. Kuhnast B, de Bruin B, Hinnen F, Tavitian B, Dollé F. Design and synthesis of a new $\left[{ }^{18} \mathrm{~F}\right]$ fluoropyridine-based haloacetamide reagent for the labeling of oligonucleotides: 2-bromo-N-[3-(2-[ $\left.{ }^{18} \mathrm{~F}\right]$ fluoropyridin-3-yloxy)propyl] acetamide. Bioconjug Chem. 2004;15:617-627.

\section{Terry Jones}

The PET Research Advisory Company

8 Prestbury $R d$.

Wilmslow, Cheshire, SK9 2LJ U.K.

E-mail: terry.jones40@googlemail.com

\section{Pat Price}

Department of Surgery and Cancer

Imperial College Healthcare NHS Trust

Hammersmith Hospital

Du Cane Rd.

London, U.K.

\section{Bertrand Tavitian}

Service Hospitalier Frédéric Joliot

$C E A, D S V, I^{2} B M$

Orsay, France

DOI: 10.2967/jnumed.111.094920 